EPJ Web of Conferences 110,01026 (2016)

DOI: $10.1051 /$ epjconf/201611001026

(C) Owned by the authors, published by EDP Sciences, 2016

\title{
MODELING OF HYDRODYNAMICS OF A HIGHLY CONCENTRATED GRANULAR MEDIUM ON THE BASIS OF A POWER-LAW
}

\author{
Alexander Shvab ${ }^{1,}$, Natalia Kagenova ${ }^{1}$ \\ ${ }^{1}$ National Research Tomsk State University, 634050 Tomsk, Russia
}

\begin{abstract}
The paper deals with the movement of the granular medium at a high concentration on the basis of the "power" of the liquid. Based on the original partial slip boundary conditions on the walls of protection obtained with experimental and numerical data to flow in the channel at a flow obstacle.
\end{abstract}

At the moment increased interest in experimental and theoretical research of the dynamics of granular and granular medium at high concentrations. Great importance of these flows takes place in practical activity in the processing of granular media in the chemical, metallurgical, food and nuclear industries. It is known that the movement of the granular medium at a high concentration is conventionally divided into two modes. The first flow regime characterized by low shear rates, which is described by the theory of limit equilibrium [1]. The second regime corresponds to the inertial regime that meets high shear rates, which was called "the theory of rapid motions granular media" [2-3]. At the inertial regime motion of highly concentrated granular medium particles move chaotically, like molecules in a dense gas or liquid, and the stress tensor in such an environment appears due to momentum transfer of granular medium particles. The result appears the dependence of the stress tensor arising in the granular medium of the strain rate tensor. Experimental and theoretical research on the dynamics of highly granular medium in terms of rheology shows that the behavior of such a medium similar to the behavior a dilatant non-Newtonian incompressible medium. A large variety of physical properties of granular medium explains the lack of a general, rational theory of motion of highly concentrated granular medium. Therefore, at present a perspective direction is the construction of mathematical models describing the hydrodynamics this flow. In this paper we consider the motion of high-concentration granular medium motion in the inertial regime. In the scientific literature, basic direction of of theoretical research associated with establishment of relationships between internal stresses and shear rates [4-6] and no attention is paid to the boundary conditions on the solid wall.

Proposed in the work semi-empirical method for the determination of the boundary conditions on the solid surface, which takes into account not only the projection of the internal stresses on the wall, but also takes into account the effects of dry Coulomb friction between the granules granular medium and the wall [7], allowing agree the calculated and experimental data.

In constructing the physical model of the movement of the dense layer of granular medium, we assume that the highly concentrated medium is composed of solid granules, which are in mutual contact. Also the considered medium has a continuous field distribution of velocities and accelerations. In view of these assumptions in the framework mechanics non-Newtonian medium in analogy to $[8]$ can be written

$$
\tau_{i, j}=2 \mu^{n} J^{n-1} \dot{e}_{i, j}=B \dot{e}_{i, j}
$$

were $\tau_{i, j}, \dot{e}_{i, j}-$ the stress tensor and the strain rate, respectively, $\mu$ - shear viscosity, $J$ - deformation rate intensity.

For definiteness we shall consider the gravity flow of highly concentrated granular medium around the obstacle, located in a plane channel (Figure 1), for which there is the most reliable experimental data [9].

\footnotetext{
${ }^{a}$ Corresponding author: impression1994@mail.ru
} 


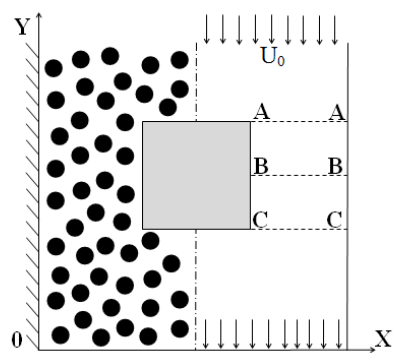

Figure 1. The study area.

On the basis of the received physical representation of a system of equations in dimensionless variables vortex - stream function describing the hydrodynamics of dense layer will be of the form

$$
\begin{gathered}
\frac{\partial \Omega}{\partial \tau}+\frac{\partial u_{x} \Omega}{\partial x}+\frac{\partial u_{y} \Omega}{\partial y}=\frac{1}{\operatorname{Re}}\left[\frac{\partial}{\partial x}\left(B \frac{\partial \Omega}{\partial x}\right)+\frac{\partial}{\partial y}\left(B \frac{\partial \Omega}{\partial y}\right)+\frac{\partial B}{\partial x} \frac{\partial \Omega}{\partial x}+\frac{\partial B}{\partial y} \frac{\partial \Omega}{\partial y}+\left(\frac{\partial^{2} B}{\partial y^{2}}-\frac{\partial^{2} B}{\partial x^{2}}\right)\left(\frac{\partial u_{y}}{\partial x}+\frac{\partial u_{x}}{\partial y}\right)+4 \frac{\partial^{2} B}{\partial x \partial y} \frac{\partial u_{x}}{\partial x}\right] \\
\frac{\partial^{2} \psi}{\partial x^{2}}+\frac{\partial^{2} \psi}{\partial y^{2}}=\Omega
\end{gathered}
$$

where

$$
\begin{gathered}
B=\left[2\left(\frac{\partial u_{x}}{\partial x}\right)^{2}+2\left(\frac{\partial u_{y}}{\partial y}\right)^{2}+\left(\frac{\partial u_{y}}{\partial x}+\frac{\partial u_{x}}{\partial y}\right)^{2}\right]^{(n-1) / 2} ; \\
\Omega=\frac{\partial u_{x}}{\partial y}-\frac{\partial u_{y}}{\partial x} ; \quad u_{x}=\frac{\partial \psi}{\partial y} ; \quad u_{y}=-\frac{\partial \psi}{\partial x} ; \quad \operatorname{Re}=\frac{\rho L^{n} U_{0}^{2-n}}{\mu^{n}} .
\end{gathered}
$$

Here Re - Reynolds number, $L$ - channel width, $U_{0}$ - scale velocity (the vertical velocity in the inlet section).

A special feature of the proposed model of the granular medium motion is a new formulation of the boundary conditions on the solid surfaces. On the wall are commonly used slip condition of medium that cause does not correspond to the theoretical and experimental data. Experimental investigations of the granular medium motion in vertical channels show that the velocity movement of particulate material on the wall does not equal zero, i.e. there is some slip of the medium on the wall. In this model, to account for the slip effect of the medium on the wall is assumed that the tangential stresses on the wall are proportional to the velocity of the medium on it to an accuracy by some constant $\beta$, which we call the coefficient of sliding. Thus, the boundary condition at the solid wall is:

$$
\frac{\partial u_{s}}{\partial n}=-\beta u_{s} ; \quad u_{n}=0
$$

where $u_{s}, u_{n}$ - the tangential and normal components of the velocity at the wall, respectively, $n$ - normal to the wall surface, $\beta$ - slip coefficient of the medium on the wall, which varies from zero (the condition of complete slip) to infinity (slip condition). It should be noted that slip coefficient $\beta$ of the medium depends on the properties of the granular medium and is determined by comparing the calculation with experiment. In the numerical solution of the problem is necessary to determine the value of a vortex and tangential velocity on the wall. Expanding the stream function in a Taylor series, and using the boundary condition (5), we find, for example, the value of a vortex on the vertical wall of the obstacles (the right half in Figure 1):

$$
\Omega_{w}=\frac{2\left(\psi_{w+1}-\psi_{w}\right)}{\Delta x^{2}} \frac{\beta}{\left(\beta+\frac{2}{\Delta x}\right)} .
$$

The speed value on the wall will

$$
\left(u_{y}\right)_{w}=-\frac{2\left(\psi_{w+1}-\psi_{w}\right)}{2 \Delta x+\beta \Delta x^{2}} .
$$


Similarly, the values of the vortex and the tangential velocity determined on the other walls.

Steady solution of (1) - (2) was an evolutionary method of establishing by time. The Poisson equation for determining the stream function (5) imagined in the form of non-stationary equation:

$$
\frac{\partial \psi}{A \partial \tau}-\left(\frac{\partial^{2} \psi}{\partial x^{2}}+\frac{\partial^{2} \psi}{\partial y^{2}}\right)=-\Omega,
$$

where the value of the constant $A$ is selected from the condition of maximum speed of the iterative process. The numerical solution was conducted using the generalized implicit scheme of variable directions. This scheme has second order accuracy in time. Convection and diffusion terms in the transport equations of eddy are written using the exponential scheme [9], which has the second order accuracy and removes the limitation of the grid Reynolds number. In the angular point two values of the vortex used in accordance with the recommendations to Roach [10].

Credibility of the proposed mathematical model (Figure 2) validated by comparing numerical and experimental data [11] for distribution the vertical velocity, depending on the coordinate $x$ in the cross sections (A-A, B-B, C-C) as shown by dotted lines in Figure 1 for the parameters $\operatorname{Re}=0.25 ; n=1.1$.

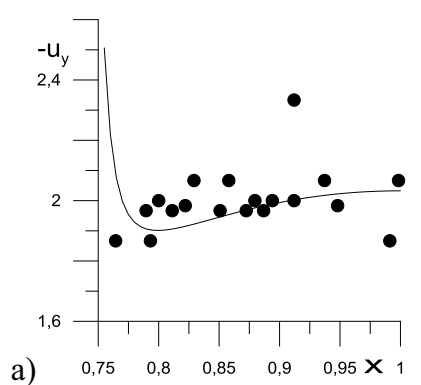

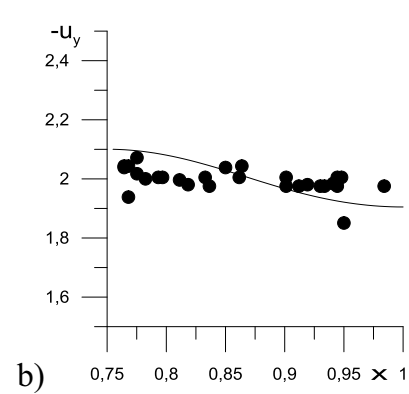

b) $\quad 0,75 \quad 0,8 \quad 0,85 \quad 0,9 \quad 0,95 \times 1$

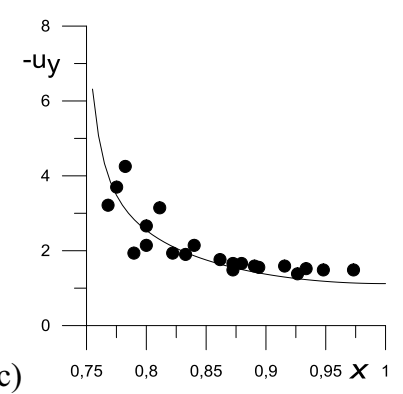

c)

Figure 2. Comparison of the obtained solution with the experimental data in the cross section shown in Figure 1: section A-A; b) section B$\mathrm{B}$, c) section C-C.

Value of the slip coefficient on the upper horizontal wall of obstacles to take to the no-slip condition $(\beta=120)$. Such a boundary condition allowed the simulate filling layer which is formed on this wall, on the basis of experimental data. On the other walls of the value of the coefficient $\beta$ was chosen equal to zero, which corresponds to the condition of full slip. It should be noted that a more realistic formulation of the boundary conditions on the walls can more accurately agree on the experimental and calculated data. The degree indicator of nonlinearity $n$ of highly concentrated granular medium rather weakly differs from the value corresponding to the model of Newtonian viscous fluid.

The work is supported by RFBR (The Russian Foundation for Basic Research) grant 13-08-00372-a.

\section{References}

1. Geniev G.A. The dynamics of the granular medium (M.: Gosstroiizdat, 1958)

2. I.V.Shirko, The mechanics of granular media: Theory of fast motions. Collection of papers (M.: Mir, 1985)

3. Hutter K., Rajagopal K.R. ,Contiuum Mech / Thermodyn., 6, 81 (1994)

4. Savage S.B., Jeffrey D.J. The stress tensor in a granular flow at high shear rates (M.: Mir, 1985)

5. Jenkins J.T., Savage S.B., J.Fluid Mech., 130, 187 (1983)

6. Yu. A. Berezin, L.A. Spodareva, Journal of Applied Mechanics and Technical Physics, 39 (2), 117 (1998)

7. Shvab A.V., Martsenko M.S., Ryzhih Y.N., Engineer-nat. magazine, 84 (4), 676 (2011)

8. Shulman Z.P., Convective heat transfer rheologicaly complex fluids (M.: Energia, 1975)

9. Nedderman R., S. Davies and D. Norton, The Flow of Granular Materials Round Obstacles (The mechanics of granular media: Theory of fast motions. Collection of papers, M.: Mir, 1985).

10. Patankar C., Numerical methods for solving problems of heat transfer and fluid dynamics (M.: Energoatomizdat, 1984)

11. P. Roach. Computational fluid dynamics (M.: Mir, 1980) 